\title{
Battling CRISPR-Cas9 off-target genome editing
}

\author{
Daisy Li • Hong Zhou (iD) - Xiao Zeng
}

Received: 9 June 2019 / Accepted: 10 July 2019/Published online: 16 July 2019

(C) Springer Nature B.V. 2019

CRISPR-Cas9 has become a widely adopted tool for gene editing and gene expression suppression/activation. First discovered in bacteria as a defensive mechanism against phage infection, CRISPR-Cas9 can also be introduced in mammalian system since the DNArepairing mechanisms are evolutionally conserved throughout all organisms. The real attraction of CRISPR-Cas9 is that the mutation can be pre-designed and targeted to specific locations anywhere in the genome, making gene editing "programmable". The core of CRISPR-Cas9 system is composed of the Cas9 endonuclease and a single-guide RNA (sgRNA) with a 20base user-defined spacer sequence. This sgRNA can lead Cas9 nuclease to any a genomic locus that both matches the spacer sequence and has a protospacer adjacent motif (PAM) immediately downstream. That said, limitations apply. The sgRNA is prone to leading the Cas9 to genomic loci sharing sequence similarity with the sgRNA spacer. Therefore, off-target effect or the elimination of which has become the major huddle for transforming this valuable discovery into a reliable

D. $\mathrm{Li}$

Department of Anthropology, Emory University, Atlanta, GA 30322, USA

\section{H. Zhou $(\bowtie)$}

Department of Mathematical Sciences, University of Saint Joseph, West Hartford, CT 06117, USA

e-mail: hzhou@usj.edu

\section{Zeng $(\bowtie)$}

PBSG, LLC, 905 W 7th St., Frederick, MD 21701, USA

e-mail: xiao.zeng@planbscientific.com weapon for fighting human diseases.

It is understood that Cas 9 off-target nuclease activity is likely a result of the evolutional arms-race between bacteria and viruses. Bacteria that survived virus infection store characteristic virus genetic sequences as spacers which can be transcribed into RNA to guide the Cas nuclease to degrade virus genetic elements containing the same sequence. This process provides bacteria an adaptive immune system to resist repeated phage invasion. Since phages' genetic sequence inherently mutates, some of these variations would escape Cas nuclease attack. In return, the bacterial CRISPRCas system evolves by allowing Cas nuclease to target sequences sharing certain degrees of homology with the spacer sequence.

Off-target genome editing not only can introduce uncertainty into scientific discoveries about gene functions, but also confounds and emasculates the therapeutic applications of CRISPR-Cas9. All the strategies to battle off-target genome editing can be grouped into five categories, namely (1) computational and mathematical predication, (2) experimental off-target cleavage validation, (3) Cas9-sgRNA delivery modification, (4) highfidelity SpCas9 engineering, and (5) guide RNA engineering.

The early computational methods to predict potential genome off-target loci were built upon the data collected using SpCas9, the most commonly used type II CRISPR-associated nuclease (Fu et al. 2013; Hsu et al. 2013; Jinek et al. 2012; Lin et al. 2014; Pattanayak et al. 2013; Tsai et al. 2015). Though some details of these findings contradict with each other, a consensus has 
been reached: (1) Off-target effects decrease when the number of mismatches (including both base mismatches and bulges) between sgRNA and target sequence increases; (2) Cas9 is less tolerant of mismatches proximal to PAM. Later methods were able to achieve better predication because they incorporated new knowledge of Cas9 domains, especially the related energetics parameters. (Alkan et al. 2018; Klein et al. 2018; Xu et al. 2017; Young et al. 2019; Zhang et al. 2019). In 2017, using an innovative computer algorithm, we scanned the entire protein-coding regions of human genome for best sgRNA designs (Zhou et al. 2017). Among almost two million sgRNA designed, only two can pass a stringent off-target filter. However, if we exclude off-target loci outside gene sequences and take into consideration the weaker activity of the secondary PAMs, $89 \%$ proteincoding genes can have at least 1 and 54\% can have at least 10 designed sgRNAs free of potential off-target loci.

Computational tools can exhaust all possible offtarget sites, but such predictions must be further validated experimentally since a large portion of predicted or identified in vitro off-target sequences do not cause in vivo cleavages (Fu et al. 2013; Hsu et al. 2013; Pattanayak et al. 2013). Whole genome sequencing is the most comprehensive approach to detect off-target mutations, but its high cost makes it impractical. Both GUIDE-Seq and DISCOVER-Seq provide a reliable and efficient alternative for genome-wide off-target cleavage detection based on next generation sequencing, but the two methods apply different techniques to capture DNA fragments generated from double-strand breaks. (Tsai et al. 2015; Wienert et al. 2019). For an even more cost-effective validation, we have created a searchable database of PCR primer pairs that span the potential off-target cleavage sites inside protein-coding sequences across human genome (www.pbsgweb.com).

The third strategy is based on the speculation that in a cell, there is only one Cas9-sgRNA complex that can occupy the target and additional complexes can only bind off-target substrates. Thus, limiting either the Cas9 or sgRNA concentration, or both, can reduce off-target effect. This hypothesis is supported by several studies (Fu et al. 2013; Hsu et al. 2013; Tsai et al. 2015). In another attempt, Kim et al. managed to premix Cas9 protein and sgRNA to form a complex (RNA complex) in vitro. Then the RNP complex is delivered directly into mammalian cells (Kim et al. 2014). The fact that RNP is rapidly degraded in cells, the authors claimed, contributes to the achieved higher specificity. Taking a different approach, Davis and collaborators fused a protein into Cas 9 to disrupt its activity such that its nuclease function is activated only in the presence of a small molecule. They found that this inducible Cas9 system can reduce off-target effect at the expense of some ontarget efficacy (Davis et al. 2015).

The double nicking technique employs two different Cas9 nickases, each engineered to break a separate DNA strand. Both nickases must be simultaneously present and proximal to each other to achieve a pseudo double-stranded DNA break. Since it is unlikely to have two off-target sites near each other, double nicking is the first method that greatly minimizes off-target genome editing (Cho et al. 2014; Ran et al. 2013), though it also significantly reduces the on-target efficacy. That said, a recent publication reported unexpected on-target mutagenesis in applying this method, raising safety concern (Alateeq et al. 2018). Another line of rational design of high-fidelity SpCas9 aims to reduce the energetics of the interaction between Cas9-sgRNA complex and its target DNA by introducing amino substitution(s) into the Cas 9 protein. The first two high-fidelity SpCas9 mutants are eSpCas9 (Slaymaker et al. 2016) and SpCas9-HF1 (Kleinstiver et al. 2016), both can significantly improve target specificity and remain robust on-target cleavage. They cannot, however, completely abolish genomewide off-target activities, which is motivating the creation of other high-fidelity SpCas9 variants including HypaCas9, HeFSpCas9, xCas9, SpCas9-NG, EvoCas9, and Sniper-Cas9 (Casini et al. 2018; Chen et al. 2017; $\mathrm{Hu}$ et al. 2018; Kulcsár et al. 2017; Lee et al. 2019; Nishimasu et al. 2018). Among them, SpCas9-NG can recognize NG PAMs and xCas9 can recognize NG, GAA, and GAT PAMS. These two variants broaden the DNA-targeting spectrum.

Engineering guide RNAs provide another feasible strategy. Using 5'-truncated sgRNA can improve specificity but would also undermine the on-target effect ( $\mathrm{Fu}$ et al. 2014). Kocak et al. extended the 5'-end of sgRNA spacer to obtain hp-sgRNAs that can form a short hairpin structure at the $5^{\prime}$ terminal (Kocak et al. 2019). The hairpin structure elevates the energetics requirement for R-loop formation which is a critical step for DNA cleavage. Since RNA-DNA mispairing at off-target sites bears mitigated energetics, hp-sgRNAs can significantly increase sgRNA-Cas9 specificity. In another example, Ryan et al. chemically modified the ribose-phosphate backbone at selected sites of the 20-base guide RNA 
spacer and found that the modified sgRNAs can reduce off-target effects (Ryan et al. 2018). The increased specificity is likely contributed by the lowered stability between guide RNA and mismatched off-target DNA sequences.

The search continues. It seems that no single strategy discussed in this review can win the battle by itself, although high-fidelity Cas 9 mutants might have been the most promising. It is becoming clear in our point of view that a combination of these strategies, for instance, a combination of computational modeling backed by repeated validation, and high-fidelity Cas9s, may soon remove the uncertainty incurred by off-target genome editing.

\section{References}

Alateeq S, Ovchinnikov D, Tracey T, Whitworth D, Al-Rubaish A, Al-Ali A, et al. Identification of on-target mutagenesis during correction of a beta-thalassemia splice mutation in iPS cells with optimised CRISPR/Cas9-double nickase reveals potential safety concerns. APL Bioengineering. 2018;2:1-18.

Alkan F, Wenzel A, Anthon C, Havgaard JH, Gorodkin J. CRISPR-Cas9 off-targeting assessment with nucleic acid duplex energy parameters. Genome Biol. 2018;19:177. https://doi.org/10.1186/s13059-018-1534-x.

Casini A, Olivieri M, Petris G, Montagna C, Reginato G, Maule G, et al. A highly specific SpCas9 variant is identified by in vivo screening in yeast. Nat Biotechnol. 2018;36(3):265-71.

Chen JS, Dagdas YS, Kleinstiver BP, Welch MM, Sousa AA, Harrington LB, et al. Enhanced proofreading governs CRISPR-Cas 9 targeting accuracy. Nature. 2017;550:40710.

Cho SW, Kim S, Kim Y, Kweon J, Kim HS, Bae S, et al. Analysis of off-target effects of CRISPR/Cas-derived RNA-guided endonucleases and nickases. Genome Res. 2014;24:132-41.

Davis KM, Pattanayak V, Thompson DB, Zuris JA, Liu DR. Small molecule-triggered Cas9 protein with improved genomeediting specificity. Nat Chem Biol. 2015;11(5):316-8.

Fu Y, Foden JA, Khayter C, Maeder ML, Reyon D, Joung JK, et al. High frequency off-target mutagenesis induced by CRISPR-Cas nucleases in human cells. Nat Biotechnol. 2013;31:822-6.

Fu Y, Sander JD, Reyon D, Cascio VM, Joung JK. Improving CRISPR-Cas nuclease specificity using truncated guide RNAs. Nat Biotechnol. 2014;32:279-84.

Hsu PD, Scott DA, Weinstein JA, Ran FA, Konermann S, Agarwala V, et al. DNA targeting specificity of RNAguided Cas9 nucleases. Nat Biotechnol. 2013;31:827-32.

Hu JH, Miller SM, Geurts MH, Tang W, Chen L, Sun N, et al. Evolved Cas 9 variants with broad PAM compatibility and high DNA specificity. Nature. 2018;556:57-63.

Jinek M, Chylinski K, Fonfara I, Hauer M, Doudna JA, Charpentier E. A programmable dual-RNA-guided DNA endonuclease in adaptive bacterial immunity. Science. 2012;337:816-21.

Kim S, Kim D, Cho SW, Kim J, Kim JS. Highly efficient RNAguided genome editing in human cells via delivery of purified Cas9 ribonucleoproteins. Genome Res. 2014;24:1012-9.

Klein M, Eslami-Mossallam B, Arroyo DG, Depken M. Hybridization kinetics explains CRISPR-Cas off-targeting rules. Cell Rep. 2018;22:1413-23.

Kleinstiver BP, Pattanayak V, Prew MS, Tsai SQ, Nguyen N, Zheng Z, et al. High-fidelity CRISPR-Cas9 variants with undetectable genome-wide off-targets. Nature. 2016;529: 490-5.

Kocak DD, Josephs EA, Bhandarkar V, Adkar SS, Kwon JB, Gersbach CA. Increasing the specificity of CRISPR systems with engineered RNA secondary structures. Nat Biotechnol. 2019;37:657-66.

Kulcsár PI, Tálas A, Huszár K, Ligeti Z, Tóth E, Weinhardt N, et al. Crossing enhanced and high fidelity SpCas9 nucleases to optimize specificity and cleavage. Genome Biol. 2017;18: 190.

Lee J, Jung MH, Jeong E, Lee JK. Using sniper-Cas9 to minimize off-target fffects of CRISPR-Cas9 without the loss of ontarget activity via directed evolution. JOVE. 2019;144: e59202.

Lin Y, Cradick TJ, Brown MT, Deshmukh H, Ranjan P, Sarode N, et al. CRISPR/Cas9 systems have off-target activity with insertions or deletions between target DNA and guide RNA sequences. Nucleic Acids Res. 2014;42:7473-85.

Nishimasu H, Shi X, Ishiguro S, Gao L, Hirano S, Okazaki S, et al. Engineered CRISPR-Cas9 nuclease with expanded targeting space. Science. 2018;361:1259-62.

Pattanayak V, Lin S, Guilinger JP, Ma E, Doudna JA, Liu DR. High-throughput profiling of off-target DNA cleavage reveals RNA-programmed Cas9 nuclease specificity. Nat Biotechnol. 2013;31:839-43.

Ran FA, Hsu PD, Lin CY, Gootenberg JS, Konermann S, Trevino AE, et al. Double nicking by RNA-guided CRISPR Cas9 for enhanced genome editing specificity. Cell. 2013;154:13809.

Ryan DE, Taussig D, Steinfeld I, Phadnis SM, Lunstad BD2, Singh $\mathrm{M}$, et al. Improving CRISPR-Cas specificity with chemical modifications in single-guide RNAs. Nucleic Acids Res. 2018;46:792-803.

Slaymaker IM, Gao L, Zetsche B, Scott DA, Yan WX, Zhang F. Rationally engineered Cas 9 nucleases with improved specificity. Science. 2016;1(351):84-8.

Tsai SQ, Zheng Z, Nguyen NT, Liebers M, Topkar VV, Thapar V, et al. GUIDE-Seq enables genome-wide profiling of offtarget cleavage by CRISPR-Cas nucleases. Nat Biotechnol. 2015;33:187-97.

Wienert B, Wyman SK, Richardson CD, Yeh CD, Akcakaya P, Porritt MJ, et al. Unbiased detection of CRISPR off-targets in vivo using DISCOVER-Seq. Science. 2019;364:286-9.

$\mathrm{Xu}$ X, Duan D, Chen SJ. CRISPR-Cas9 cleavage efficiency correlates strongly with target sgRNA folding stability: from physical mechanism to off-target assessment. Sci Rep. 2017;7:143.

Young J, Zastrow-Hayes G, Deschamps S, Svitashev S, Zaremba M, Acharya A, et al. CRISPR-Cas9 editing in maize: systematic evaluation of off-target activity and its relevance in crop improvement. Sci Rep. 2019;9:6729. 
Zhang D, Hurst T, Duand D, Chen SJ. Unified energetics analysis unravels SpCas9 cleavage activity for optimal gRNA design. PNAS. 2019; 116:8693-8.

Zhou H, Zhou M, Li D, Manthey J, Lioutikova E, Wang H, et al. Whole genome analysis of CRISPR Cas9 sgRNA off-target homologies via an efficient computational algorithm. BMC Genomics. 2017;18(Suppl 9):826.
Publisher's note Springer Nature remains neutral with regard to jurisdictional claims in published maps and institutional affiliations. 